\section{The bone marrow leaves its scar: new concepts in pulmonary fibrosis}

\author{
Sarah E. Dunsmore and Steven D. Shapiro \\ Respiratory and Critical Care Medicine, Brigham and Women's Hospital, \\ Harvard Medical School, Boston, Massachusetts, USA
}

Excess collagen deposition occurs in pulmonary fibrosis. A new study suggests that collagen overproduction may originate from cells derived from bone marrow precursors rather than parenchymal lung fibroblasts (see the related article beginning on page 243 ).

J. Clin. Invest. 113:180-182 (2004). doi:10.1172/JCI200420782.

Idiopathic pulmonary fibrosis (IPF) is a devastating disorder with no effective treatment, which insidiously advances to airspace obliteration and death. The mean survival time following diagnosis is less than 5 years. Pathologically, the disease is characterized by chronic inflammation and exuberant collagen production within the lung (1). Survival can be predicted by the extent of fibroblastic foci (Figure 1) present at the time of lung biopsy (2). While the role of inflammation in IPF is less clear, inflammatory mediators, particularly TGF- $\beta$, are presumed to drive the fibrotic process. Myofibroblasts, a distinguishing feature of fibroblastic foci (3), are thought to arise from local activation of parenchymal fibroblasts by TGF- $\beta$ and are historically considered to be the primary collagen-producing cell in fibrotic lesions $(3,4)$.

In this issue of the JCI, Hashimoto and colleagues (5) strongly force us to reconsider these fundamental concepts regarding the origin and phenotype of pathologic fibroblasts. In

Address correspondence to: Steven D. Shapiro, Respiratory and Clinical Care Medicine, Brigham and Women's Hospital, 75 Francis Street, Boston, Massachusetts 02115, USA. Phone: (617) 732-7599; Fax: (617) 232-4623;

E-mail: sshapiro@rics.bwh.harvard.edu. Conflict of interest: The authors have declared that no conflict of interest exists. Nonstandard abbreviations used: idiopathic pulmonary fibrosis (IPF); stromal cell-derived factor-1 (SDF-1); secondary lymphoid tissue chemokine (SLC). this study, the authors experimentally induced pulmonary fibrosis via bleomycin administration to mice that had been reconstituted with bone marrow derived from GFP transgenic mice. Thus, in the reconstituted mice, cells derived from bone marrow precursors will express GFP, and resident cells will not. Most of the collagen-producing fibroblasts observed in the lungs of these mice were of bone marrow rather than of local origin. Interestingly, GFP-positive fibroblasts could not be induced by TGF- $\beta$ to express $\alpha$-smooth muscle actin. Therefore, the pathologic collagen-producing fibroblasts not only were bone marrow-derived but were incapable of transforming into myofibroblasts.
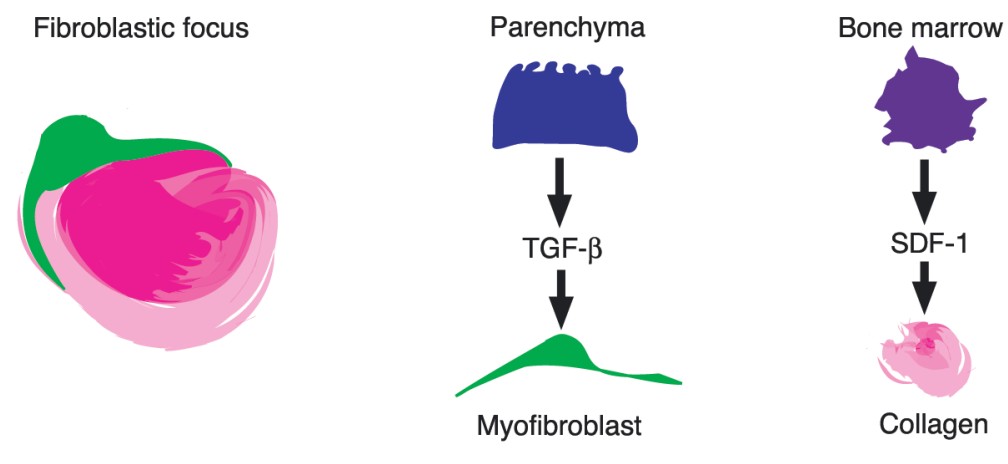

Figure 1

Origin of fibroblastic foci. Fibroblastic foci, sites of active collagen synthesis, are the pathologic hallmark of pulmonary fibrosis. The traditional view is that cytokine secretion by epithelial and inflammatory cells induces parenchymal lung fibroblasts to overproduce collagen and to differentiate into myofibroblasts. Several recent studies indicate that bone marrow-derived cells can engraft into lung tissue and produce collagen. Bone marrow-derived cells may be recruited to the lung by chemokines generated by macrophages. 

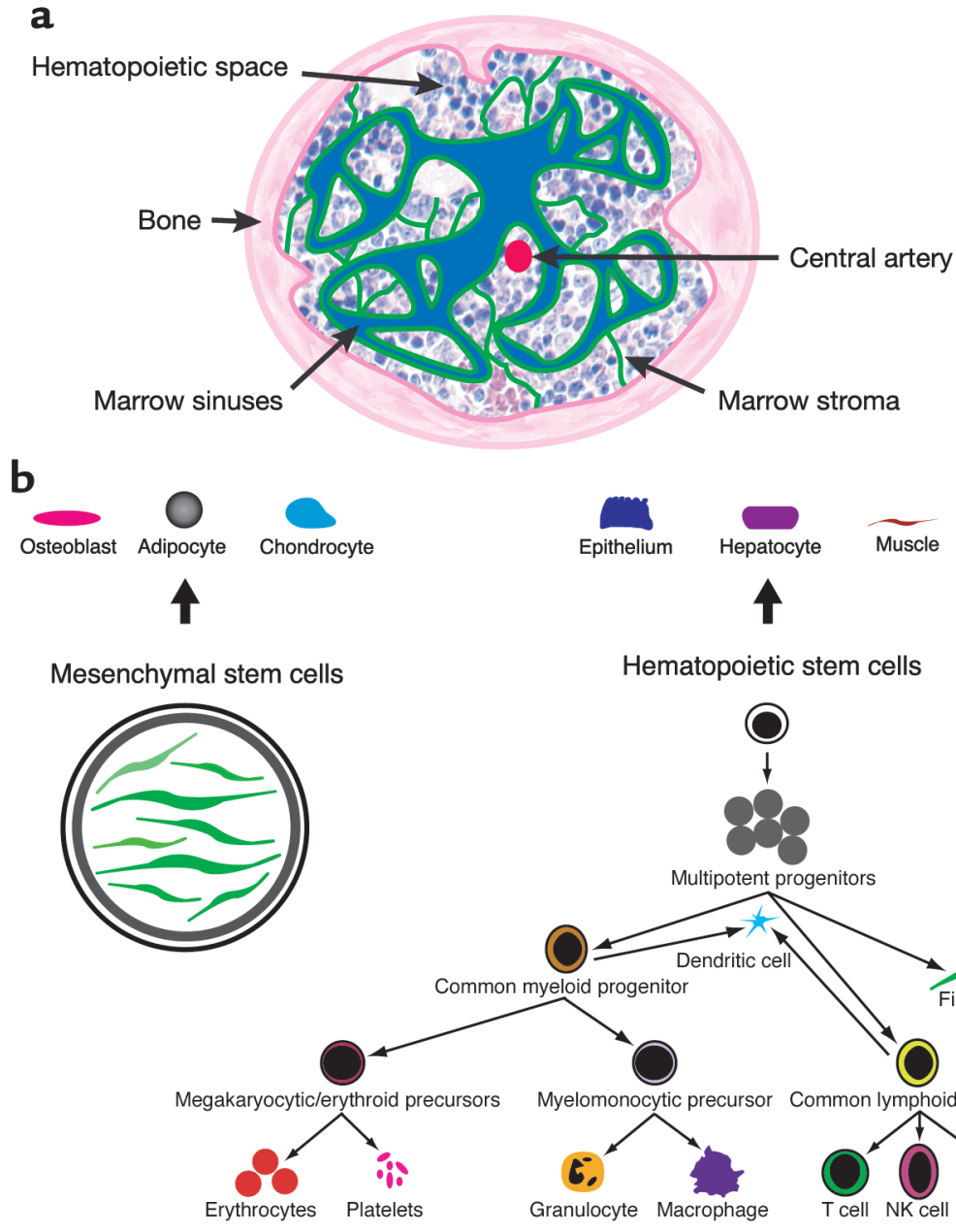

Hematopoietic stem cells

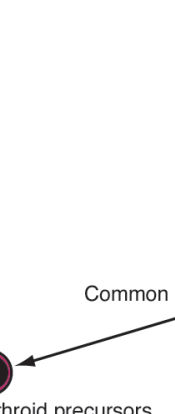

\section{.}
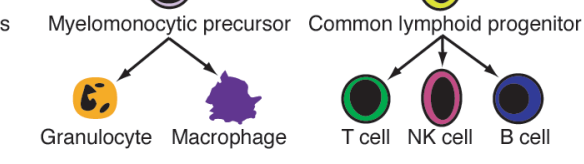

Studies have shown that bone marrow-derived stromal cells (7) and hematopoietic precursors (8) engraft and become structural cells, including fibroblasts, particularly following tissue injury. In fact, a unique population of collagen-expressing cells derived from hematopoietic precursors termed "fibrocytes" has been described $(9,10)$ and is likely the bone marrow cell population from which the GFP-positive cells in the lungs of bleomycin-treated mice originate. Moreover, fibrocytes express the chemokine receptors CXCR4 and CCR7 and migrate in vitro in response to their corresponding ligands, stromal cell-derived factor-1 (SDF-1) and secondary lymphoid-tissue chemokine (SLC) (10). Hashimoto et al. demonstrated that levels of SDF-1 and SLC are increased in the lung following bleomycin treatment (5), indicating that these chemokines also may be the in vivo signals that recruit bone marrow- derived fibroblasts to the lung. In another study of radiation-induced fibrosis (11), influx of bone marrow-derived macrophages preceded that of bone marrow-derived fibroblasts, leading the authors to speculate that these macrophages may be the source of SDF-1, SLC, or other substances that recruit fibroblast precursors from the bone marrow.

Other investigators have systemically administered mesenchymal stem cells to bleomycin-treated mice (12, 13). In these studies, cells that engrafted into lung tissue displayed epithelial characteristics. The expression of epithelial markers by GFP-positive cells was not assessed by Hashimoto et al. (5), but it is intriguing to speculate that the GFP-positive, collagen-producing cells underwent epithelial-mesenchymal transition (14). Alternatively, the isolation and culture of bone marrow cells prior to reconstitution may have predisposed them to an

\section{Figure 2}

Bone marrow cell populations. (a) Cross section of tubular bone showing the structure of bone marrow. Hematopoietic spaces are organized into cords by stromal cells (green lines) and contain distinct foci of developing erythroid and granulocytic cells interspersed among immature hematopoietic precursors. Mature blood cells enter the circulation by migrating across the marrow sinus endothelium. (b) Two populations of stem cells may be isolated from bone marrow. Mesenchymal stem cells, isolated from marrow stro$\mathrm{ma}$, adhere to tissue culture plasticware and have been shown to have the potential to differentiate into osteoblasts, adipocytes, and chondrocytes. Hematopoietic stem cells, which do not adhere to tissue culture plasticware, not only have the capacity to produce mature blood cells but also have been shown to engraft into liver, lung, kidney, heart, skeletal muscle, pancreas, and gastrointestinal tract. Fibrocytes, defined by the cell surface expression of collagen I, CD11b, CD13, CD34, CD45RO, MHC class II, and CD86, express the same chemokine receptors, CXCR4 and CCR7, as the collagen I-positive, GFP-positive cells in bleomycininduced fibrotic lesions.

epithelial phenotype. In any event, understanding mechanisms of engraftment will be important as clinical applications of bone marrow stem cell therapy are explored.

\section{Potential for stem cell therapy in the lung}

Finally, Phan et al. (5) should be particularly congratulated because they had previously provided critical data (4) lending support to the previous paradigm that is now contested. Scientific truth is an elusive concept that we hope to achieve as new techniques are utilized and fresh hypotheses tested. Often we shift directions and must revise or abandon concepts once cherished. Although the fibroblast clearly remains a pathologic player in fibrosis (for now), its origin and properties must be reevaluated. The clinical implications of these findings are significant; for example, we might now consider bone marrow stem cell therapy to correct structural alter- 
ations in the lung. Translation of our understanding of disease pathogenesis into clinical practice will bring us closer to our real goal - improving the lives of our patients and ultimately curing disease. With the publication of this study, IPF patients may someday breathe more easily.

\section{Acknowledgments}

The authors thank Jeffery Kutok for providing the mouse bone marrow sections used in Figure 2.

1. Gross, T.J., and Hunninghake, G.W. 2001. Idiopathic pulmonary fibrosis. N. Engl. J. Med. 345:517-525.

2. King, T.E., Jr., et al. 2001. Idiopathic pulmonary fibrosis: relationship between histopathologic features and mortality. Am.J.
Respir. Crit. Care Med. 164:1025-1032.

3. Kuhn, C., and McDonald, J.A. 1991. The roles of the myofibroblast in idiopathic pulmonary fibrosis. Ultrastructural and immunohistochemical features of sites of active extracellular matrix synthesis. Am. J. Pathol. 138: $1257-1265$.

4. Zhang, K., Rekhter, M.D., Gordon, D., and Phan, S.H. 1994. Myofibroblasts and their role in lung collagen gene expression during pulmonary fibrosis. A combined immunohistochemical and in situ hybridization study. Am. J. Pathol. 145:114-125.

5. Hashimoto, N., Jin, H., Liu, T., Chensue, S.W., and Phan, S.H. 2004. Bone marrow-derived progenitor cells in pulmonary fibrosis. J. Clin. Invest. 113:243-252. doi:10.1172/JCI200418847.

6. Schrier, D.J., Kunkel, R.G., and Phan, S.H. 1983. The role of strain variation in murine bleomycin induced pulmonary fibrosis. Am. Rev. Respir. Dis. 127:63-66.

7. Prockop, D.J. 1997. Marrow stromal cells as stem cells for nonhematopoietic tissues. Science. 276:71-74.

8. Herzog, E.L., Chai, L., and Krause, D.S. 2003.
Plasticity of marrow-derived stem cells. Blood 102:3483-3493.

9. Bucala, R., Spiegel, L.A., Chesney, J., Hogan, M. and Cerami, A. 1994. Circulating fibrocytes define a new leukocyte subpopulation that mediates tissue repair. Mol. Med. 1:71-81.

10. Abe, R., Donnelly, S.C., Peng, T., Bucula, R., and Metz, C.N. 2001. Peripheral blood fibrocytes: differentiation pathway and migration to wound sites. J. Immunol. 166:7556-7662.

11. Epperly, M.W., Guo, H., Gretton, J.E., and Greenberger, J.S. 2003. Bone marrow origin of myofibroblasts in irradiation pulmonary fibrosis. $\mathrm{Am}$. J. Respir. Cell Mol. Biol. 29:213-224.

12. Kotton, D.N., et al. 2001. Bone marrow-derived cells as progenitors of lung alveolar epithelium. Development. 128:5181-5188.

13. Ortiz, L.A., et al. 2003. Mesenchymal stem cell engraftment in lung is enhanced in response to bleomycin exposure and ameliorates its fibrotic effects. Proc. Natl. Acad. Sci. U. S. A 100:8407-8411.

14. Iwano, M., et al. 2002. Evidence that fibroblasts derive from epithelium during tissue fibrosis. J. Clin. Invest. 110:341-350. doi:10.1172/JCI200215518.

\title{
Bateman domains and adenosine derivatives form a binding contract
}

\author{
Bruce E. Kemp ${ }^{1,2}$ \\ ${ }^{1}$ St. Vincent's Institute of Medical Research, Fitzroy, Victoria, Australia \\ ${ }^{2}$ Commonwealth Scientific and Industrial Research Organisation (CSIRO) \\ Health Sciences and Nutrition, Parkville, Victoria, Australia
}

\begin{abstract}
Conserved pairs of CBS sequence motifs (named after cystathionine $\beta$-synthase) found in a wide variety of proteins associate to form Bateman domains. A new study establishes that Bateman domains bind adenosyl compounds and regulate IMP dehydrogenase, CBS, chloride channels, and AMP-activated protein kinase (see the related article beginning on page 274). This discovery reveals how mutations in CBS sequences in these proteins cause hereditary diseases and provides a rich vista of conceptual opportunities for therapies in energy metabolism, obesity, diabetes, cancer, antivirals, and immunosuppression.
\end{abstract}

J. Clin. Invest. 113:182-184 (2004). doi:10.1172/JCI200420846.

\begin{abstract}
What are CBS sequence motifs?
Alexander Bateman first recognized cystathionine $\beta$-synthase (CBS) sequences by looking for internal sequence duplications within proteins of the Methanococcus janaschii genome. These CBS se-
\end{abstract}

Address correspondence to: Bruce E. Kemp, St. Vincent's Institute of Medical Research, 41 Victoria Parade, Fitzroy, Victoria 3065, Australia. Phone: 61-2-9288-2480;

Fax: 61-3-9416-2676;

E-mail: kemp@ariel.unimelb.edu.au.

Conflict of interest: The author has declared that no conflict of interest exists.

Nonstandard abbreviations used:

cystathionine $\beta$-synthase (CBS); IMP

dehydrogenase (IMPDH); chloride channel

(CLC); AMP-activated protein kinase (AMPK). quences, typically 60 residues in length, occur as tandem pairs in a diversity of proteins, from archaebacteria to eukaryotes $(1,2)$, including IMP dehydrogenase (IMPDH), whose protein crystal structure shows that CBS sequence pairs form a discrete structural domain (3) termed a Bateman domain (Figure 1) (4). Although it is known that mutations in CBS sequences alter the control functions of their parent proteins and cause hereditary diseases (5-7), the precise mechanisms involved were unknown. Mutations in CBS cause homocystinuria; IMPDH mutations cause retinitis pigmentosa; mutations in chloride channels (CLC1, CLC2, CLC5, and
CLCKB) cause a variety of conditions, including congenital myotonia, idiopathic generalized epilepsy, hypercalciuric nephrolithiasis, and classic Bartter syndrome; mutations in the AMP-activated protein kinase (AMPK) $\gamma 2$ subunit cause cardiac conductance problems similar to Wolff-Parkinson-White syndrome; and mutations in Hampshire pig $\gamma 3$ cause skeletal muscle glycogen storage disease.

\section{Bateman domains bind adenosyl compounds}

In this issue of the JCI, Scott and colleagues (8) have now found the missing link by showing that pairs of CBS sequences derived from AMPK, IMPDH-2, the chloride channel CLC2, and CBS bind adenosyl compounds (Figure 1). They show that AMPK, with its two pairs of CBS sequences, binds two molecules of AMP or ATP; the single pair of CBS sequences present in IMPDH allows it to bind a single mole of ATP, as does the pair of CBS sequences in CLC2. CBS binds a single mole of $S$-adenosyl methionine. The authors introduced known hereditary mutations into CBS sequences of the parent protein and found that they caused dramatic shifts in ligand-binding properties, which explained the loss of allosteric control of the parent protein. These findings will also influence research into the wider family of CBScontaining proteins (2) that includes other ion transporters, hemolysin, and poly (A) polymerase. 